\title{
AVALIAÇÃO DO IMPACTO DA RESOLUÇÃO ESPACIAL DE MODELOS DIGITAIS DE ELEVAÇÃO SOBRE A ESTIMATIVA DE FORMAÇÃO DE OZÔNIO SUPERFICIAL USANDO MODELS-3
}

\author{
LINDOLFO MEIRA ${ }^{1}$, JORGE RICARDO DUCATI ${ }^{1}$ E ELBA CALESSO TEIXEIRA ${ }^{2}$ \\ ${ }^{1}$ Universidade Federal do Rio Grande do Sul/ Centro Estadual de Pesquisas em Sensoriamento Remoto e \\ Meteorologia (UFRGS/CEPSRM), Porto Alegre - RS, Brasil \\ ${ }^{2}$ Fundação Estadual de Proteção Ambiental (FEPAM), Porto Alegre - RS, Brasil \\ meira@if.ufrgs.br,ducati@if.ufrgs.br. \\ gerpro.pesquisa@fepam.rs.gov.br
}

Recebido Setembro 2008 - Aceito Junho 2009

\begin{abstract}
RESUMO
Este trabalho estabelece uma comparação básica entre dois cenários distintos de simulação da formação de ozônio superficial sobre o Estado do Rio Grande do Sul. No primeiro cenário usa-se um modelo digital de elevação com resolução de 9 km com a interface de simulação "Models-3". No segundo cenário, um modelo com resolução de $3 \mathrm{~km}$ é usado. Em cada cenário, implementa-se um único domínio de simulação, com resoluções correspondentes as do respectivo modelo. Dados do Centro Nacional Estadunidense de Previsão Ambiental foram usados na simulação meteorológica, e dados do Instituto Brasileiro de Geografia e Estatística e do Departamento de Trânsito do Rio Grande do Sul foram usados na estimativa das emissões devidas à frota veicular da Região Metropolitana de Porto Alegre (única fonte emissora aqui considerada). Os resultados apresentaram diferenças marcantes entre um cenário e outro. As concentrações constantemente elevadas de ozônio observadas à resolução de $9 \mathrm{~km}$, tiveram seus níveis reduzidos à metade a $3 \mathrm{~km}$. Também os padrões de dispersão tiveram características bem distintas, espalhando-se amplamente sobre a parte sul do domínio de simulação no primeiro cenário e, no segundo, espalhando-se de forma mais limitada sobre a parte sudoeste, com um jato observado em direção noroeste. Elevados níveis de concentração de ozônio foram observados a cerca de $500 \mathrm{~km}$ das fontes emissoras.
\end{abstract}

Palavras-Chave: MDE, MQA, Ozônio, Models-3.

\begin{abstract}
EVALUATION OF THE IMPACT OF DIGITAL ELEVATION MODEL RESOLUTION OVER THE ESTIMATIVE OF SURFACE OZONE FORMATION USING MODELS-3

This study establishes a basic comparison between two different simulation scenarios of surface ozone formation over the Rio Grande do Sul State. In the first simulation scenario, a $9 \mathrm{~km}$ resolution digital elevation model is used within the so-called Models-3 simulation interface. In the second one, a 3 $\mathrm{km}$ resolution model is used. For each scenario, a single simulation domain was implemented, with resolutions matching the respective model. US National Center for Environmental Prediction's data were used to simulate meteorology, as well as the Brazilian Institute for Geography and Statistics and the Rio Grande do Sul Traffic Department's data were used to estimate emissions by the Metropolitan Area of Porto Alegre vehicle fleet, supposed as the only source of the emissions. The results present striking differences between the two scenarios: the sustained high ozone concentrations values observed at $9 \mathrm{~km}$ resolution dropped to half at $3 \mathrm{~km}$ resolution. Also, dispersion patterns had quite distinguishable features, spreading widely over the south part of the simulation domain in the first scenario and spreading in a narrower scheme over the south-west region, with a jet observed northwestwards. High levels of ozone concentration were observed as far as $500 \mathrm{~km}$ from the emission sources.
\end{abstract}

Keywords: DEM, AQM, Ozone, Models-3. 


\section{INTRODUÇÃO}

A despeito do fato de que estratégias de controle da qualidade do ar venham sendo implementadas há muito tempo, o problema da poluição atmosférica persiste. Entre os tópicos de maior interesse nas questões de poluição atmosférica, o ozônio $\left(\mathrm{O}_{3}\right)$ tem ocupado atualmente posição de destaque. Altos índices de concentração de ozônio superficial (ozônio no nível do solo) têm sido causa de grande preocupação nos centros urbanos em todo o mundo (Amann, 2001). Isto se deve, sobretudo, aos efeitos deste gás sobre a saúde humana e a vegetação. Tais efeitos, no entanto, não se restringem somente às áreas urbanas: a síntese do ozônio é governada por processos não-lineares (Kleinman, 2005), assim como acontece com sua dispersão e de seus precursores. Isto faz com que altos índices de concentração deste gás sejam observados mesmo em lugares remotos, onde a produção por emissões locais seria desprezível (Manahan, 1979).

No intuito de abordar de forma cada vez mais precisa todos os aspectos relacionados à síntese e à dispersão do ozônio, modelos numéricos têm sido empregados regularmente nos últimos anos. Alguns dos, assim chamados, modelos de qualidade do ar (MQA), sobretudo os empregados no estudo de atmosferas urbanas, baseiam-se em simplificações de modelos sofisticados (Sojhi et al., 2006) e, ao passo em que fornecem soluções bastante rápidas, não descrevem a contento a totalidade dos processos e interações que conduzem o comportamento cinético-químico dos poluentes atmosféricos. No caso do ozônio, cuja síntese e dispersão derivam de processos extremamente complexos, uma descrição precisa é particularmente importante.

Anova geração de modelos atmosféricos, aproveitando-se enormemente do insaturável desenvolvimento dos computadores pessoais e das aplicações destes ao processamento paralelo, aborda hoje o problema da formação e dispersão química de poluentes atmosféricos de forma integrada, resolvendo sistemas de equações diferenciais parciais através de métodos avançados de cálculo numérico. A idéia da implementação de um modelo integrado de ampla abrangência, e capaz de simular sinergeticamente todos os processos envolvidos na síntese e dispersão de poluentes atmosféricos, vem sendo desenvolvida desde o início da década passada e culminou, em 1998, no lançamento do que hoje é conhecido como a interface de modelagem atmosférica Models-3 (Dennis et al., 1996). A interface é composta, primordialmente, por três módulos base: o de simulação da circulação atmosférica e assimilação altimétrica, conduzido pela $5^{a}$ Geração do Modelo de Mesoescala (MM5); o de processamento de emissões atmosféricas, conduzido pelo Operador Cerne de Emissões a Matrizes Esparsas (SMOKE); e o de simulação da dispersão química, conduzido pelo Modelo Comunitário Multiescalar de Qualidade do Ar (CMAQ).
O MM5 é um sistema hidrodinâmico, montado sobre coordenadas verticais conformes à estrutura altimétrica da área modelada, a qual pode ser limitada à ordem de escalas urbanas, e que foi originalmente desenvolvido para simular e/ou prognosticar a circulação atmosférica de mesoescala (Grell et al., 1994). No caso do SMOKE, o propósito é o de converter dados de inventários de emissões a resoluções necessárias aos modelos de qualidade do ar: inventários de emissões atmosféricas são tipicamente construídos com base em valores anuais de emissão para cada tipo de fonte, ao passo em que os modelos de qualidade do ar, geralmente necessitam de valores horários de emissões para cada uma das espécies químicas modeladas (Houyoux et al., 2000). Já o CMAQ constitui um poderoso sistema de modelagem e avaliação da qualidade do ar que vem sendo regularmente utilizado em trabalhos de caráter científico, bem como em aplicações de apoio à implementação de políticas ambientais (Byun e Ching, 1999). O modelo é capaz de simular, simultaneamente, processos de síntese troposférica de ozônio, deposição ácida, formação de particulados e outros poluentes atmosféricos (tanto em escala regional quanto em escala urbana). O modelo é fundamentado no conceito básico de atmosfera única, no qual somente a simulação da ação concorrente do conjunto de todos os processos físicos e químicos da atmosfera é que constitui elemento capaz de conferir precisão às simulações (Tao et al., 2005).

O intuito primordial deste estudo é o de avaliar a resposta da interface Models-3 na simulação horária da síntese e dispersão de ozônio superficial frente, única e exclusivamente, à mudança da estrutura altimétrica da região alvo; neste caso particular, o estado do Rio Grande do Sul (RS). O estudo compreende um período de 48 horas (desde 3 horas do dia 31/3/2006 até 3 horas do dia $2 / 4 / 2006$, coordenadas UTC), no qual são conduzidas simulações da formação e dispersão de ozônio em dois cenários distintos: no primeiro, a altimetria da região é horizontalmente resolvida em $9 \mathrm{~km}$; no segundo, em $3 \mathrm{~km}$. As diferenças entre estes dois cenários são mínimas quando comparadas às diferenças entre a melhor resolução suportada pelos modelos da interface Models-3, que é de $1 \mathrm{~km}$, e a melhor resolução dos modelos digitais de elevação (MDEs) publicamente disponíveis hoje em dia, que chega a $30 \mathrm{~m}$ (Valeriano, 2004). O tempo total das simulações foi de 60 horas, observandose um período transiente de 12 horas a fim de minimizar os efeitos das condições iniciais sobre os resultados finais.

Ao passo em que uma série de estudos têm sido conduzidos na tentativa de verificar a sensibilidade de modelos de qualidade do ar frente a diferentes parametrizações (Mao et al., 2006), pouco tem sido discutido acerca da influência da altimetria - ou, diga-se, dos MDEs - sobre os resultados finais de simulações da qualidade do ar. O raciocínio óbvio leva à conclusão de que quanto melhor a resolução do MDE 
empregado, mais precisos os resultados das simulações. No entanto, a utilização de MDEs de alta resolução em modelos de qualidade do ar tem enorme custo computacional, o que torna importante o conhecimento da relação entre a resolução dos MDEs e a fidelidade das simulações. Idealmente, o que se deseja é o equilíbrio entre eficiência computacional (devida à resolução do MDE empregado) e precisão nas previsões. A avaliação aqui feita visa atentar para a necessidade de estudos mais detalhados sobre o tema, expondo o fato de que mesmo pequenas mudanças na representação altimétrica de uma região, podem levar um mesmo modelo de simulação da qualidade do ar a registrar resultados significativamente distintos.

\section{DADOS E MÉTODOS}

\subsection{Circulação Atmosférica}

O MDE utilizado nas simulações foi derivado de dados da Shuttle Radar Topographic Mission, ou SRTM (Valeriano, 2004). Ao passo em que a resolução vertical do modelo SRTM foi mantida, a resolução horizontal foi adaptada à resolução dos domínios de simulação: no primeiro cenário definiu-se o domínio com um ponto central de referência em $30,5^{\circ} \mathrm{S}$ e $53,65^{\circ} \mathrm{W}$ e dimensão de $90 \times 90$ células com $9 \times 9 \mathrm{~km}$; no segundo, utiliza-se o mesmo ponto de referência e dimensão de $270 \times 270$ células com $3 \times 3 \mathrm{~km}$. Verticalmente, o domínio foi definido em 24 níveis (coordenadas $\sigma$ ): 1 (na superfície); 0,99; 0,$98 ; 0,96 ; 0,93 ; 0,89$; e de 0,85 até 0 , variando em intervalos de 0,05 . O nível $\sigma=0$ corresponde ao limite vertical superior de pressão, fixado em 10 mbar. O programa TERRAIN (Guo e Chen, 1994), que é um pré-processador, ou módulo, do MM5, é o responsável pela assimilação dos dados altimétricos (que foram, posteriormente, transmitidos aos outros módulos da interface de modelagem). A Figura 1 mostra a configuração horizontal do domínio no primeiro cenário. Dos arquivos que formam o MDE SRTM, utilizou-se neste trabalho o arquivo W060S10, que cobre todo o sul do Brasil e pequenas partes de países vizinhos. $\mathrm{O}$ arquivo pode ser obtido através do endereço eletrônico ftp://e0srp01u.ecs.nasa.gov.

Dois arquivos de saída foram gerados pelo TERRAIN, um para cada domínio de simulação. Estes arquivos, somados aos arquivos referentes às análises meteorológicas (obtidos através do NCEP, National Center for Environmental Prediction), contêm todos os dados necessários ao programa REGRID, que é um outro módulo do MM5. O REGRID tem a função de interpretar as análises meteorológicas nos vários níveis de pressão e interpolar tais análises ao domínio de simulação definido no TERRAIN.

$\mathrm{Na}$ simulação da circulação atmosférica durante o período de estudo (período que foi escolhido por conta da estabilidade das condições sinóticas e disponibilidade de dados de entrada para o modelo), o MM5 foi parametrizado da seguinte forma: microfísica de nuvens, algoritmo gelo simples (Grell et al., 1994); CLP, algoritmo de Hong-Pan, também dito esquema MRF-PBL (Troen e Mahrt, 1986); radiação atmosférica, esquema Nuvem-Radiação (Grell et al., 1994); temperatura do solo, algoritmo FLSS (Guo e Chen, 1994). O arquivo de saída do MM5 representa a dinâmica da troposfera sobre a área do estudo, no particular intervalo de tempo em que este se deu, e serviu como dado de entrada aos outros dois modelos da interface Models-3 usados subseqüentemente, o SMOKE e o CMAQ.

\subsection{Emissões Atmosféricas}

Somente emissões atmosféricas veiculares foram aqui consideradas, e somente aquelas devidas à frota automotiva da Região Metropolitana de Porto Alegre (RMPA). A abordagem dita VMT (Vehicular Miles Travelled) foi a empregada neste estudo. Nesta abordagem, um inventário de atividade veicular e dados meteorológicos são fornecidos ao SMOKE e, assim, o modelo estima as emissões. No inventariamento da atividade veicular na RMPA, foram considerados apenas os veículos movidos à gasolina e diesel. $\mathrm{O}$ inventário foi construído por meio de estatísticas simples, levando em consideração o tamanho da frota veicular (1.123.803 veículos movidos à gasolina e 84.925 movidos a diesel, segundo dados fornecidos pelo DETRANRS) e a extensão da malha viária (EMV) da RMPA, calculada a partir do mapa vetorial dos setores censitários brasileiros, disponibilizado pelo Instituto Brasileiro de Geografia e Estatística (IBGE) através do endereço eletrônico ftp://geoftp. ibge.gov.br. O cálculo resultou em uma malha com $6.021,79 \mathrm{~km}$ de extensão. Na distribuição espacial das emissões utilizou-se um mapa da rede municipal brasileira, disponibilizado também pelo IBGE, através do mesmo endereço eletrônico. Acerca da distribuição temporal, adotou-se um perfil com distribuição aproximadamente gaussiana para cada espécie resultante, para cada dia simulado: taxas de emissão variando de hora em hora com picos às 14 horas de cada um dos dois dias. As espécies resultantes, a saber, foram o $\mathrm{CO}, \mathrm{NO}, \mathrm{NO}_{2}, \mathrm{COV}, \mathrm{PSO}_{4}$ (sulfato agregado a material particulado), $\mathrm{PM}_{10}$ e $\mathrm{PM}_{2,5}$.

O SMOKE gerou um único arquivo de saída para cada um dos cenários de simulação (cada qual cobrindo todo o intervalo de tempo da simulação). Estes arquivos representam, em linhas gerais, a dinâmica das emissões veiculares atmosféricas na RMPA, no particular intervalo de tempo deste estudo e, junto com os arquivos de saída do MM5, serviram como dado de entrada ao CMAQ.

\subsection{Síntese e Dispersão de Ozônio}


A simulação da síntese e dispersão de ozônio foi feita em quatro etapas. A exemplo do MM5, o modelo CMAQ também é composto por módulos. Na primeira das quatro etapas o módulo JPROC foi utilizado para calcular as taxas das reações de fotodissociação (fotólise) na atmosfera no particular período de tempo do estudo. O mecanismo químico adotado nestas simulações foi o CB-IV (Yarwood et al., 2005), e os dados necessários para o cálculo das taxas de fotólise foram extraídos de valores padrão fornecidos pelo próprio modelo, de acordo com o período da simulação e a localização geográfica de seus domínios (WMO, 1986). As taxas de fotólise calculadas foram gravadas em arquivos ASCII (um arquivo para cada dia simulado) e serviram como referência cruzada durante a execução de módulos subseqüentes. É fácil notar que estas taxas não têm nenhuma relação com nenhum dado altimétrico; logo, o módulo JPROC foi acionado uma única vez e os resultados, então gerados, foram usados nas simulações de ambos os cenários.

Modelos de qualidade do ar são formulados com base em equações diferenciais ordinárias e parciais, cujas soluções necessitam de condições iniciais e de contorno. Assim, na segunda etapa da modelagem da dispersão fotoquímica o módulo ICON foi utilizado para estabelecer estas condições iniciais, que no caso específico das equações diferenciais de modelos de qualidade do ar, melhor seriam descritas como concentrações iniciais. Os dados necessários ao cálculo foram repassados ao módulo através de tabelas ASCII contendo valores padrão de concentração utilizados pelo modelo, ajustados conforme os dados de concentração fornecidos pela FEPAM. Dois arquivos de saída foram gerados, um para cada cenário, com as condições iniciais necessárias, definidas em todas as células dos respectivos domínios de simulação. Não há diferenças operacionais entre a segunda e terceira etapas, exceto pelo fato de que o módulo então utilizado foi o BCON, que estabelece condições de contorno ao invés de condições iniciais. Ou seja, os arquivos de saída gerados contêm dados de concentração definidos somente nas células da borda dos domínios de simulação.

Na quarta etapa, tomando como dado de entrada os dados gerados nas três etapas anteriores, além dos dados de saída dos modelos MM5 e SMOKE, a simulação da formação e dispersão do ozônio foi feita através do módulo CCTM. A parametrização deste módulo diz respeito à escolha dos algoritmos de simulação dos processos de transporte atmosférico, química e dinâmica de nuvens. Acerca dos processos de transporte atmosférico das espécies químicas, utilizou-se neste estudo o algoritmo de Yamartino-Blackman (Yamartino, 1993), apropriado tanto para advecção quanto difusão. Nos processos relacionados à

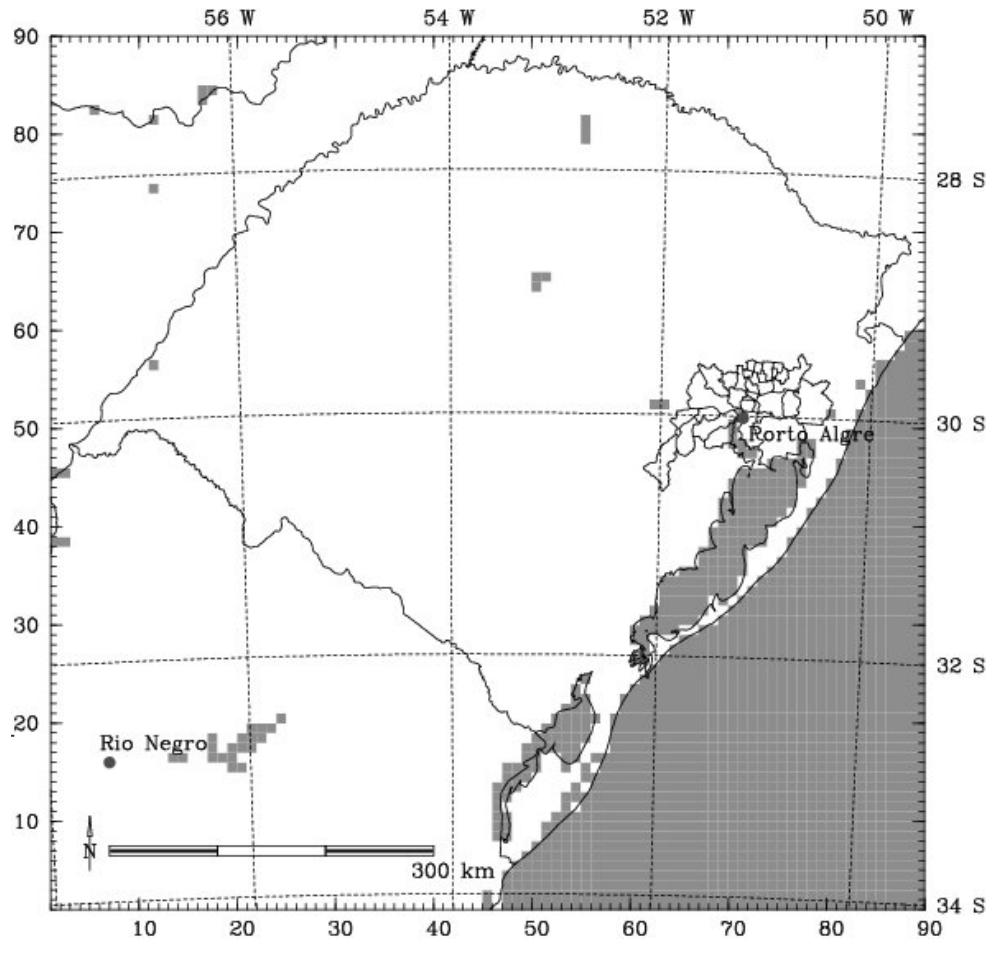

Figura 1 - Domínio de simulação assimilado no primeiro cenário. Em destaque, dentro da RMPA, a cidade de Porto Alegre e, no Uruguai, a cidade de Rio Negro. 
dinâmica de nuvens e a reações químicas por elas ocasionadas, o algoritmo ACM (Pleim e Chang, 1992) foi utilizado. Os arquivos de saída do módulo CCTM contêm os resultados finais das simulações do CMAQ; ou seja, os resultados das concentrações calculadas sobre todo espaço tridimensional do domínio de simulação, para cada espécie química estudada, bem como as taxas de remoção troposférica (por deposição seca e úmida) destas espécies. A análise feita sobre estes resultados, no entanto, refere-se ao ozônio superficial avaliado sobre os pontos geográficos que representam os centros das cidades de Porto Alegre (fonte das emissões) e de Rio Negro, no Uruguai (destino primário do ozônio formado durante o particular período destas simulações).

\section{RESULTADOS E DISCUSSÃO}

Comparando as médias horárias das concentrações simuladas na cidade de Porto Alegre, nota-se certa similaridade no padrão de variação da concentração em função do tempo, embora os valores absolutos obtidos no segundo cenário, tenham sido consistentemente mais elevados do que no primeiro cenário (Figura 2). Neste último caso, picos de concentração ocorrem entre 7-19 h e 31-48 h, com valores máximos atingindo, aproximadamente, $30 \mathrm{ppbV}$ em $15 \mathrm{~h}$ do primeiro intervalo e 82 ppbV e $105 \mathrm{ppbV}$ em, respectivamente, 38 e $42 \mathrm{~h}$ do segundo intervalo. No segundo cenário, os picos de concentração acontecem, praticamente, nos mesmos intervalos e os valores máximos observados ocorrem em 15 e $17 \mathrm{~h}$, atingindo cerca de $70 \mathrm{ppbV}$, e depois em $39 \mathrm{~h}$, atingindo cerca de $140 \mathrm{ppbV}$, refletindo o fato de as taxas de formação de ozônio serem bem mais elevadas no segundo cenário do que no primeiro: no intervalo entre $7-19 \mathrm{~h}$ a diferença nas concentrações ultrapassa $100 \%$ entre o primeiro e o segundo cenário; já uma média sobre os valores observados entre $31-48 \mathrm{~h}$ mostra um aumento de cerca de $40 \%$ entre um cenário e outro.

A Figura 3 mostra a tendência geral da formação e da dispersão do ozônio, destacando os picos de concentração (marcados com uma pequena cruz nas figuras) observados em cada um dos dias simulados, nos dois cenários. O primeiro pico de concentração surge, no primeiro cenário, às $18 \mathrm{~h} \mathrm{e,} \mathrm{no}$ segundo, às $17 \mathrm{~h}$. A diferença de tempo entre os picos de emissão de precursores e os picos de concentração de ozônio, resultou na média entre os dois cenários, em 12,5 h, um valor bem próximo do que propõe a literatura (Seinfeld, 1986). Ainda acerca do primeiro pico de concentração observado, no primeiro cenário ele ocorre à cerca de $305 \mathrm{~km}$ de Porto Alegre, sobre a cidade de Herval, na fronteira com o Uruguai. No segundo cenário o pico ocorre cerca de $235 \mathrm{~km}$ da fonte emissora, sobre a cidade de Caçapava do Sul. Estas distâncias explicam-se pelo fato de que a ocorrência de picos de concentração de ozônio em função do tempo de ocorrência de picos de emissão de seus precursores depende, além de fatores ocasionais - como as condições meteorológicas - também de fatores estritamente locais (como a estrutura altimétrica): numa região altimetricamente complexa onde se estabelecesse um regime de estagnação atmosférica, os precursores se misturariam rapidamente (i.e., ainda perto da fonte) e, havendo radiação solar, formariam ozônio num intervalo de tempo mais curto; já numa região absolutamente plana, aplacada por ventos extremamente velozes, os precursores seriam carregados na atmosfera por longas distâncias (tão longas

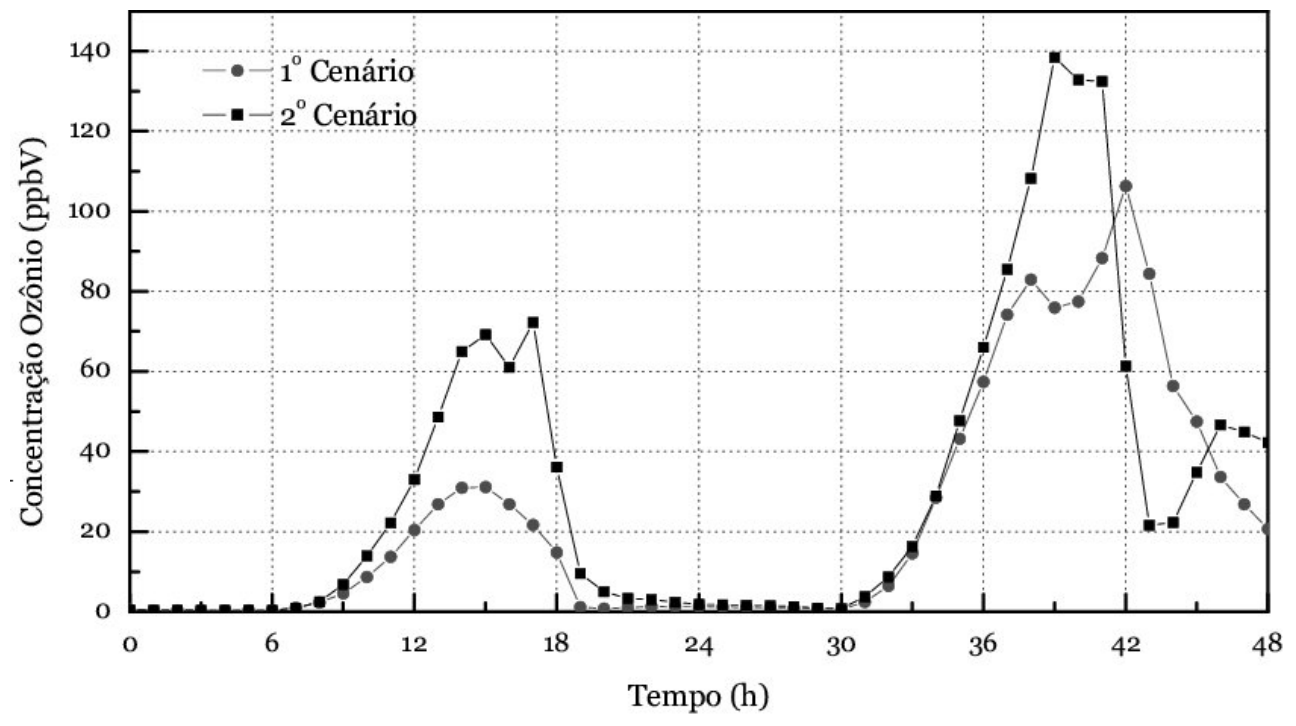

Figura 2 - Variação temporal da concentração de ozônio em Porto Alegre. 
quanto permita a velocidade do vento), dificultando a mistura e fazendo com que picos de ozônio sejam observados após um intervalo de tempo maior do que no caso anterior.

Em um segundo momento, picos de concentração de ozônio são observados, no primeiro cenário, em 39 h e, no segundo cenário, em 40 h. Nestes casos, o tempo estabelecido na literatura para a observação de picos na concentração de ozônio, em função do tempo de ocorrência dos picos de emissão dos precursores, também se aproxima muito do observado: cerca de 9,5 h na média entre o dois cenários. No caso do primeiro cenário, o pico ocorre à cerca de $66 \mathrm{~km}$ de Porto Alegre, sobre as cidades de Tapes e Barra do Ribeiro. Já no segundo cenário, o pico é observado bem próximo a Porto Alegre, ainda na RMPA, sobre as cidades de Arroio dos Ratos e São Jerônimo. Fica evidente pela Figs. 3 (b) e (d) o impacto do MDE sobre a dispersão do ozônio. No primeiro cenário, à resolução de $9 \mathrm{~km}$, as concentrações são dispersas ao Sul, e sobre uma área mais ampla (note-se a diluição nos valores de pico nas concentrações quando são comparadas as duas figuras). Já no segundo cenário, a despeito de variações mais freqüentes observadas na direção do vento, e também na maior velocidade, o padrão de escoamento é menos disperso e ocorre mais a Sudoeste, com componentes até mesmo ao Norte. É provável que a região serrana do Sudeste, melhor resolvida no segundo cenário, tenha grande impacto no comportamento observado.

A exemplo dos resultados analisados sobre a cidade de Porto Alegre, as médias horárias das concentrações simuladas na cidade de Rio Negro, no Uruguai, também apresentam certa similaridade no seu padrão de variação em função do tempo (Figura 4). Neste caso, no entanto, picos de concentração são observados somente no segundo dia do período de simulação, e são mais elevados no segundo cenário do que no primeiro, o que se observa pela dispersão nas Figuras 3 b e 3d, esta última mostrando que o escoamento ocorre mais a Sudoeste, daí os picos mais elevados em Rio Negro no segundo cenário. Os valores máximos observados nas concentrações atingem cerca de 270 ppbV em $41 \mathrm{~h}$ ( $17 \mathrm{~h}$ do segundo dia do período simulado) no segundo cenário. Já no primeiro, que ocorre uma hora mais cedo, o valor máximo não atinge os 160 ppbV. Ainda acerca deste cenário, observa-se uma ligeira mudança, em 32 h, na taxa de formação de ozônio, que se torna negativa até 34 h, o que não acontece no segundo cenário. Também a exemplo do que ocorreu em Porto Alegre, há um aumento de cerca de 100\% nas concentrações observadas entre um cenário e outro, exceto, é claro, pelo primeiro dia de simulação. E comparando-se o primeiro e o segundo dia de simulação em quaisquer cenários,
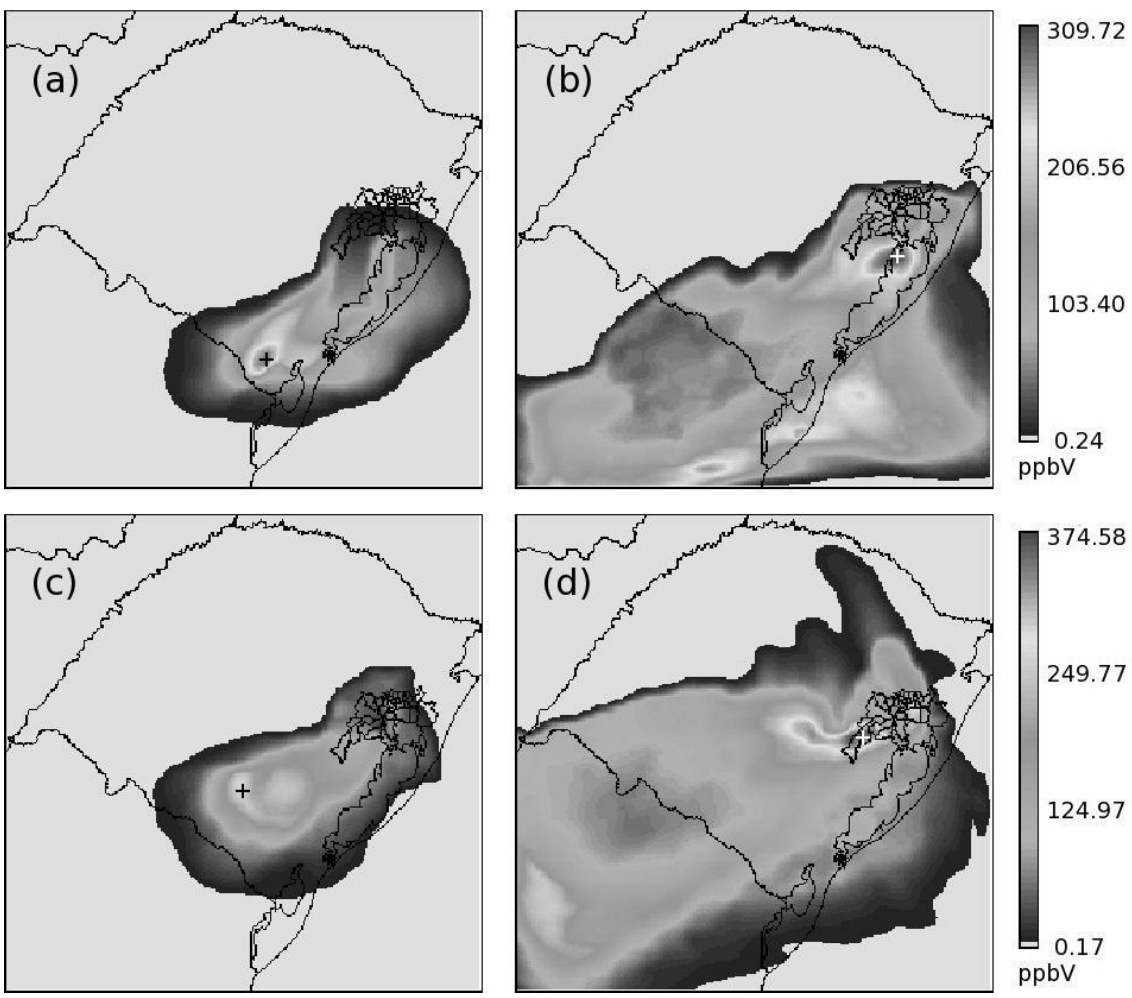

Figura 3 - Evolução na dispersão de ozônio sobre o domínio de simulação: (a) $1^{\circ}$ cenário, $1^{\circ}$ dia de simulação, $18 \mathrm{~h}$ LST; (b) $1^{\circ}$ cenário, $2^{\circ}$ dia, $18 \mathrm{~h}$ LST; (c) $2^{\circ}$ cenário, $1^{\circ}$ dia, $17 \mathrm{~h}$ LST; (d) $2^{\circ}$ cenário, $2^{\circ}$ dia, $17 \mathrm{~h} \mathrm{LST.}$ 
em vistas da ausência de picos de concentração de ozônio no primeiro dia simulado, percebe-se que o deslocamento dos precursores é bastante amplo, e que o tempo de residência destes na atmosfera, pelo menos neste caso, ultrapassou $24 \mathrm{~h}$. Ao que pareceu, atingiram em boa concentração a cidade de Rio Negro, cerca de $500 \mathrm{~km}$ da fonte emissora, fazendo com que as concentrações de ozônio fossem, pelo menos no segundo cenário, $100 \%$ mais elevadas do que em Porto Alegre.
Uma breve avaliação nas taxas de deposição seca (devido à ausência de precipitação durante o período simulado, não houve ocorrência de deposição úmida) de ozônio nos dois pontos analisados (Figuras 5 e 6), faz perceber o impacto das emissões em Porto Alegre sobre a qualidade do ar em Rio Negro (no primeiro cenário a deposição sobre Rio Negro é $505,56 \%$ maior do que em Porto Alegre; no segundo, o aumento chega a 633,33\%). A degradação na resolução do MDE, em

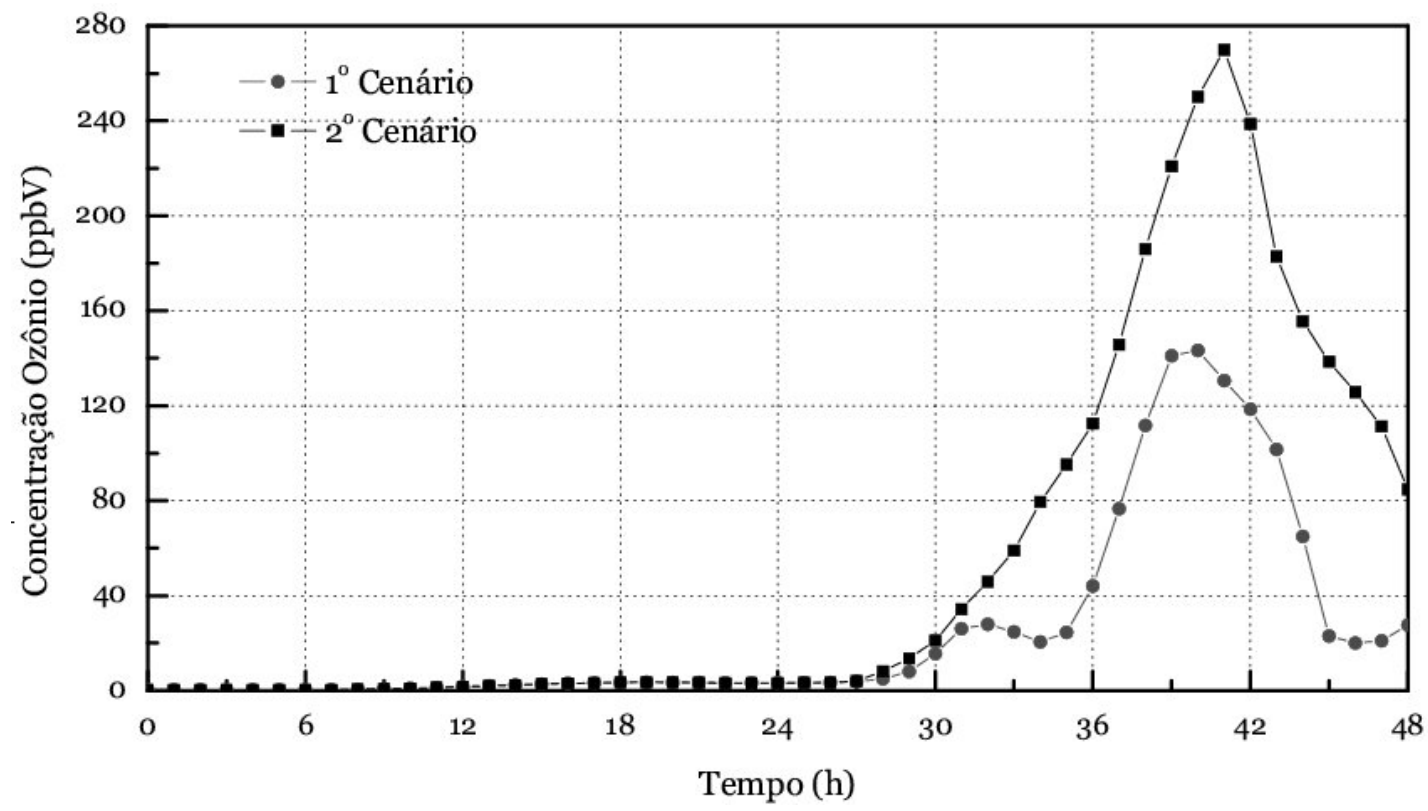

Figura 4 - Variação temporal da concentração de ozônio em Rio Negro, Uruguai.

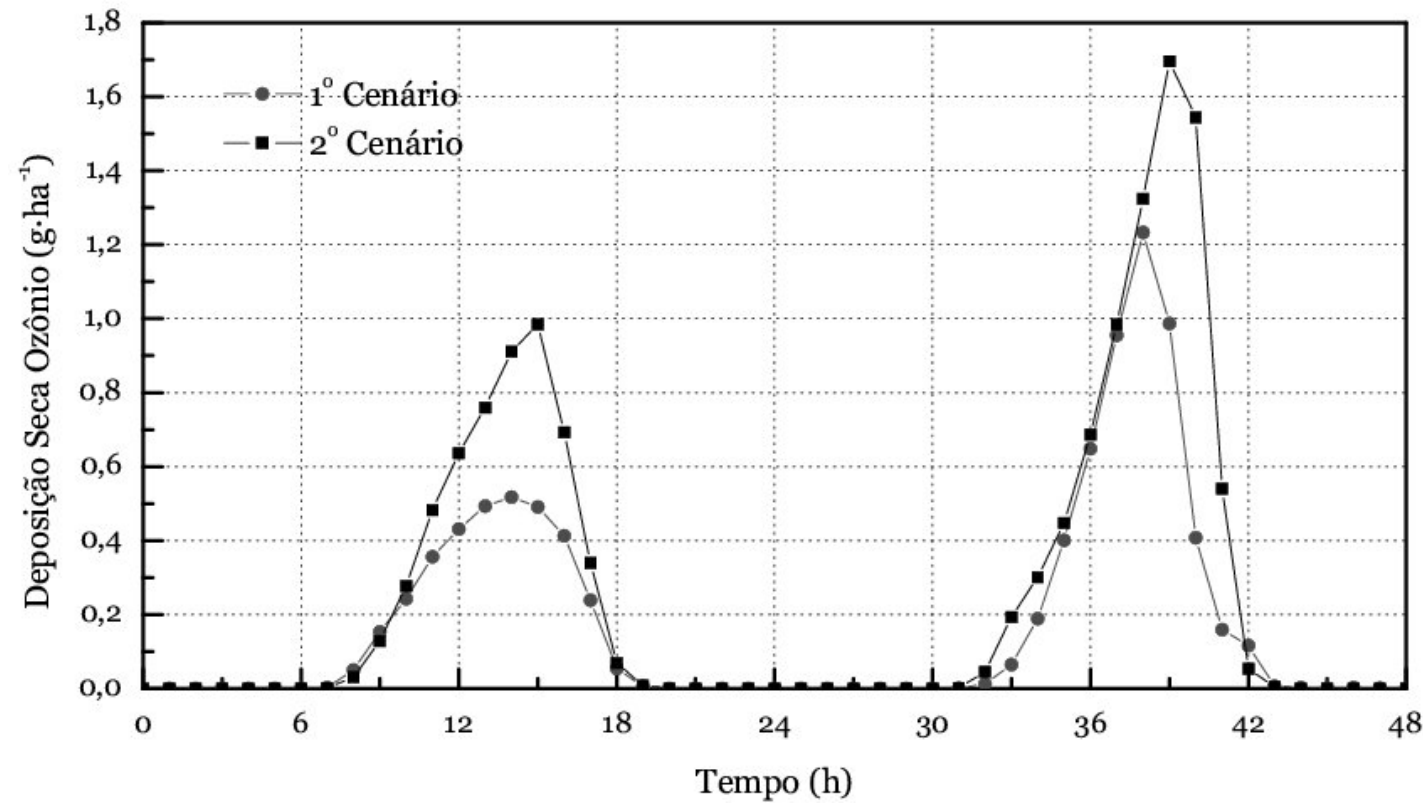

Figura 5 - Taxa de deposição seca de ozônio sobre Porto Alegre. 


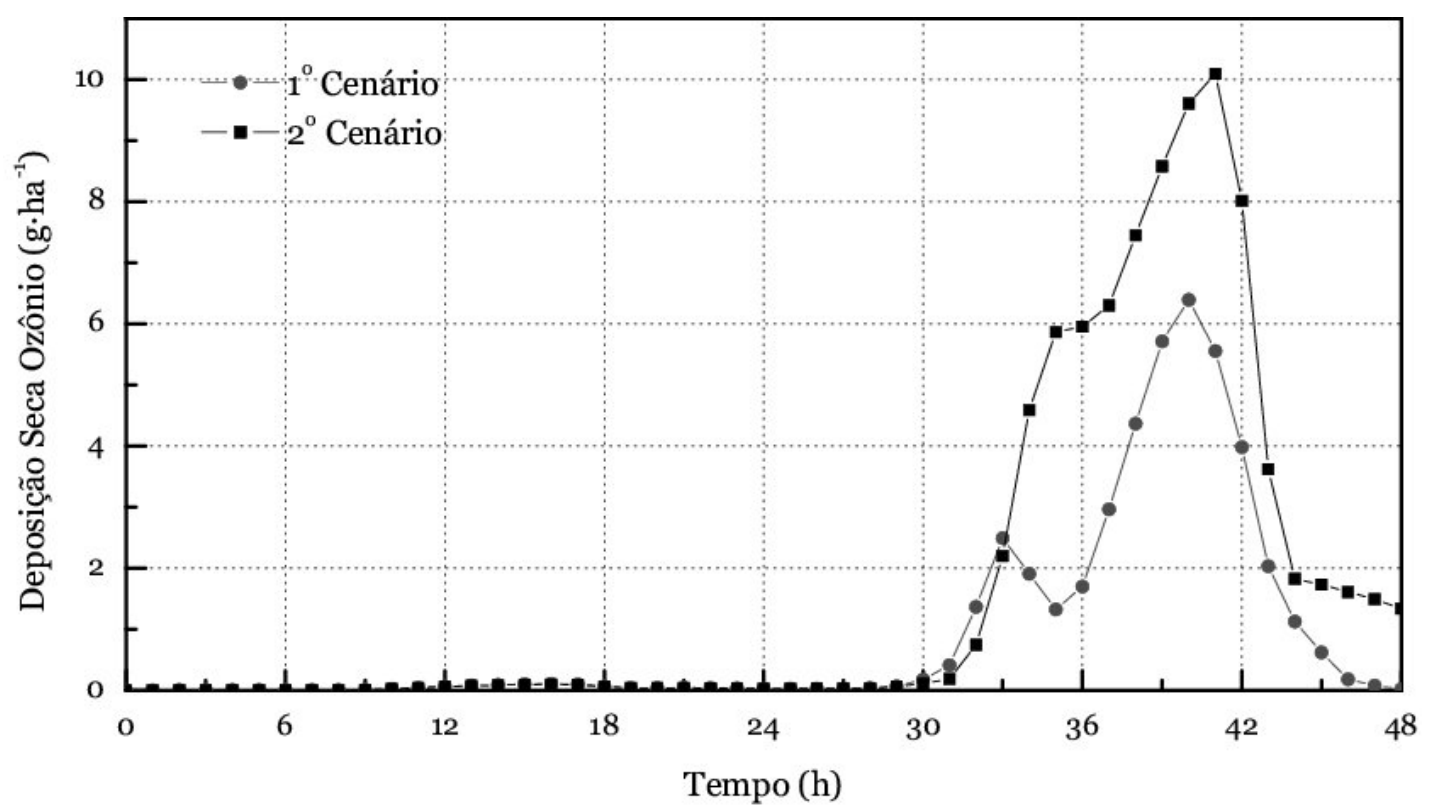

Figura 6 - Taxa de deposição seca de ozônio sobre Rio Negro, Uruguai.

geral, subestimou os valores de deposição observados (assim como o fez com as concentrações). Dividindo-se o resultado da integração temporal das curvas nos gráficos das Figuras 5 e 6 pelo intervalo total de tempo da simulação, obtém-se a concentração média do que foi depositado nos dois pontos analisados durante os dois dias simulados. A deposição média de ozônio calculada sobre Porto Alegre e Rio Negro, foi no primeiro cenário, de $0,18 \mathrm{e} 0,91 \mathrm{~g} \cdot \mathrm{ha}^{-1}$, respectivamente, e, no segundo cenário, de $0,27 \mathrm{e} 1,71 \mathrm{~g} \cdot \mathrm{ha}^{-1}$. No quadro geral, a integração espaço-temporal dos resultados obtidos para os valores de deposição seca sobre toda a área do estudo, mostrou que a deposição total, quando deteriorada a resolução, foi subestimada em $23,96 \%$.

\section{CONCLUSÕES}

O ozônio é um poluente regional, o que isenta em certo grau a ação de fontes locais de precursores sobre concentrações localmente observadas. Notou-se, pelos resultados obtidos, que os modelos digitais de elevação têm impacto significativo sobre a formação e a dispersão deste gás em simulações resultantes dos modelos numéricos da interface Models-3: a degradação na resolução do MDE neste experimento, fez diminuir à metade as concentrações simuladas de ozônio, e os padrões de dispersão também se mostraram bastante distintos. As diferenças entre os dois cenários estudados, no entanto, foram mínimas: no primeiro, a estrutura altimétrica nas simulações é resolvida em $9 \mathrm{~km}$; no segundo, a estrutura altimétrica da área estudada é resolvida em $3 \mathrm{~km}$ (foram, no entanto, mantidas as características da cobertura superficial entre os dois cenários).
Dada a natureza deste estudo, não cabe aqui a análise acerca de qual dos cenários representaria melhor a verdade de campo, mas a diferença de praticamente, $1 / 4$ entre a deposição seca no segundo cenário em relação ao primeiro aponta, em vista da leve mudança imposta à resolução do MDE utilizado, que mudanças mais drásticas deste parâmetro (como, por exemplo, simulações com $1 \mathrm{~km}$ de resolução, em contraste com $30 \mathrm{~m}$ ) podem fornecer resultados diametralmente opostos; casos nos quais uma região qualquer do território nacional, em conformidade com os padrões estabelecidos pela legislação brasileira para os níveis diários de concentração de ozônio, poderia ser taxada como fora dos padrões, e vice-versa.

Uma implementação importante ao estudo aqui apresentado seria a introdução de transições gradualmente mais intensas das características altimétricas entre os cenários, buscando-se a relação entre a resolução empregada e a precisão dos resultados das simulações. Outra implementação que, por si só, representa um tópico à parte, com conteúdo suficiente para um artigo completo, é o impacto das mudanças de resolução dos MDEs sobre as muitas variáveis simuladas pelo MM5 e as implicações sobre as simulações do CMAQ. Contudo, mais importante ainda é o fato de ficar evidente, em vista dos resultados obtidos, a necessidade de implementação de mudanças nos códigos dos modelos da interface Models-3, já que do modo como são distribuídos atualmente, não são capazes de assimilar MDEs com resoluções melhores do que $1 \mathrm{~km}$. Em MDEs com resoluções de $30 \mathrm{~m}$, por exemplo, o incremento no nível de detalhamento de estruturas altimétricas é enorme, possibilitando a análise de padrões bastante complexos em escalas muito pequenas, se comparadas às de 9 e $3 \mathrm{~km}$ aqui 
empregadas. A calibração destes modelos através de medidas de concentração de gases traço na atmosfera quer por satélites ou por redes de monitoramento in situ, também constitui área interessante de pesquisa: modelos bem calibrados poderiam ser utilizados no sentido inverso, na estimativa de inventários de emissões atmosféricas.

\section{AGRADECIMENTOS}

Este trabalho foi desenvolvido, em parte, com recursos computacionais do Centro Nacional de Supercomputação (CESUP), da Universidade Federal do Rio Grande do Sul (UFRGS).

\section{REFERÊNCIAS}

AMANN, M. Emission inventories, emission control options and control strategies: an overview of recent developments. Water, Air and Soil Pollution. Dordrecht: Kluwer, v. 130, p. 43-50, 2001.

BYUN, D. W.; CHING, J. K. S. Science algorithms of the EPA Models-3 Community Multiscale Air Quality (CMAQ) modeling system. U. S. Environmental Protection Agency Rep. EPA-600/R-99/030. Washington DC: EPA, 1999. 727 p.DENNIS, R. L. et al. The next generation of integrated air quality modeling: EPA's Models-3. Atmospheric Environment, Amsterdam, v. 30, p. 1925-1938, 1996.

GRELL, G. A.; DUDHIA, J.; STAUFFER, D. R. A description of the Fifth-Generation Penn State/NCAR Mesoscale Model (MM5). National Center for Atmospheric Research Tech. Note NCAR/TM-398+SRT. Boulder: NCAR, 1994. 138 p.

GUO, Y.; CHEN, S. Terrain and land use for fifth-generation Penn State/NCAR mesoscale modeling system (MM5): Program TERRAIN. National Center for Atmospheric Research Tech. Note, NCAR/TN-397+IA. Boulder: NCAR, 1994. 119 p.

HOUYOUX, M. R. et al. Emission inventory development and processing for the seasonal model for regional air quality (SMRQA) project. Journal of Geophysical Research, [S.I.], v. 105, n. D7, p. 9079-9090, 2000.

KLEINMAN, L. I. The dependence of tropospheric ozone production rate on ozone precursors. Atmospheric Environment, Amsterdam, v. 39, n. 10, p. 575-586, 2005.

MANAHAN, S. E. Environmental Chemistry. 3.ed. Boston: Willard Grant Press, 1979. p. 271-382.

MAO, Q. et al. Numerical experiments on MM5-CMAQ sensitivity to various PBL schemes. Atmospheric Environment, Amsterdam, v. 40, n. 12, p. 3092-3110, 2006.

PLEIM, J. E.; CHANG, J. S. A non-local closure model in the convective boundary layer Atmospheric Environment, Amsterdam, v. 26A, p. 965-981, 1992.

SOKHI, R. S. et al. Predictions of ozone levels in London using the MM5-CMAQ modelling system. Environmental Modelling \& Software, London, v. 21, p. 566-576, 2006.

SEINFELD, J. H.; PANDIS, S. N. Atmospheric chemistry and physics: from air pollution to climate change. New York: John Wiley, 1986. 738 p.

TAO, Z. et al. Area, mobile, and point source contributions to ground level ozone: a summer simulation across the continental USA. Atmospheric Environment, Amsterdam, v. 39, p. 1869-1877, 2005.

TROEN, I. B.; MAHRT, L. A simple model of the atmospheric bounday layer. Sensitivity to surface Evaporation. Boundary Layer Meteorology, [S.I.], v. 37, p. 129-148, 1986.

VALERIANO, M. M. Modelo digital de elevação com dados SRTM disponíveis para a América do Sul. Instituto Nacional de Pesquisas Espaciais, relatório técnico INPE-10550RPQ/756. São José dos Campos: INPE, 2004. 72 p.

YAMARTINO, R. J. Nonnegative, conserved scalar transport using grid-cell-centered, spectrally constrained Blackman cubics for applications on a variable-thickness mesh. Monthy Weather Reviews, [S.I.], v. 121, p. 753-763, 1993.

YARWOOD, G.; RAO, S.; YOCKE, M. 2005 Updates to the Carbon Bond chemical mechanism: CB-V. Final Report to the U.S. EPA RT-0400675, 2005.

WORLD METEOROLOGICAL ORGANIZATION. Atmospheric Ozone 1985: Assessment of Our Understanding of the Processes Controlling its Present Distribution and Change. WMO Rep. [s.n.] : Genebra, 1986. 